\title{
Análise da Plataforma de Participação Social: Participação do Cidadão e Organizações na Elaboração de Políticas Públicas
}

\author{
Vladimir Fagundes, Jonice de Oliveira Sampaio \\ Universidade Federal do Rio de Janeiro - Programa de Pós-graduação em Informática \\ (UFRJ/PPGI) \\ Térreo, Bloco E, CCMN/NCE, Cidade Universitária \\ Caixa Postal 68.530 \\ Rio de Janeiro/RJ, Brasil, 21941-590 \\ Vladimir.fagundesegmail.com, joniceddcc.ufrj.br
}

\begin{abstract}
The demand for better services, transparency and participation in public policy development, causes the Brazilian Government set actions that approach the citizen to the State. One of the actions was the construction of the Participa.br, a social network that allows the interaction of citizens and public policy. However, it is imperative to know the interactions generated by this social network. In this context, this study develops an analysis of the Participa.br to make the recognition of the elements, relationships and actors contained on the platform, in order to identify organizations with a high degree of influence on policies directed towards E-Government. The techniques of social network analysis identified the relationships between public consultations and suggestions that contributed to policy developments.
\end{abstract}

Resumo. A exigência por melhores serviços, transparência e participação na elaboração de políticas públicas, faz com que o Governo Brasileiro programe ações que aproximem o cidadão ao Estado. Uma das ações foi a construção do Participa.br, rede social que permite a interação do cidadão e políticas públicas. Porém, é imperativo conhecer as interações geradas por essa rede social. Neste contexto, este estudo elabora uma análise do Participa.br que viabiliza o reconhecimento dos elementos, relacionamentos e atores contidos na plataforma, com intuito de identificar organizações com alto grau de influências em politicas voltadas para Governo Eletrônico. As técnicas de análise de redes sociais permitiram identificar as relações entre consultas públicas e as sugestões que contribuíram para evolução das politicas.

\section{Introdução}

O termo "gestão pública participativa" tornou-se corriqueiro nos discursos políticos dos atores governamentais, porém sua prática não efetivada e o estimulo a participação do

\footnotetext{
${ }^{1}$ Gestão Pública Participativa (co-gestão pública) - são às intervenções populares, constituídas pelo conjunto de cidadãos ou associações representativas da comunidade, sobre a qual incidirão as políticas públicas, cujas demandas ganham visibilidade através das consultas populares, audiências públicas ou concerto. Aquelas intervenções têm como fim influenciar o conteúdo da
} 
cidadão é ineficiente. Disputas de interesses, precariedade de uma cultura participativa e democrática se faz notar tanto na burocracia estatal quanto nas organizações da sociedade e no setor privado, dificultam a consolidação dos espaços de diálogo e negociação sobre as políticas.

A sociedade brasileira cada vez mais clama por profissionalismo e excelência na administração pública, bem como por melhor qualidade de vida e redução das desigualdades sociais. O cidadão passa a exigir, em níveis progressivos, melhores serviços, respeito à cidadania e mais transparência e efetividade no uso dos recursos públicos. Nesse aspecto, a busca pelo aperfeiçoamento e pela profissionalização da administração pública assume papel fundamental para maximizar o desempenho do Estado brasileiro.

Tal participação gerou ações do Governo Federal no intuito de aproximar o cidadão às ações do Estado. Primeiramente, a criação do Gabinete Digital, lançado em setembro de 2013, foi anunciada como resposta às manifestações ocorridas no território brasileiro em junho do ano de 2013. A segunda medida foi a construção de um espaço digital que facilitasse que cada individuo da sociedade interagisse com as políticas públicas chamado de Portal da Participação Social (Participa.br) [BRASIL 2014]. Sendo foco de o Portal agregar as informações públicas; aprimorar a comunicação do Governo com os servidores e aperfeiçoar os canais de interação com a população.

Contudo, apesar da iniciativa de construção de um portal para participação social constituir um avanço sobre o tema "diálogo entre Entidade Pública e Sociedade", sua construção é apenas o primeiro passo para que a formulação de políticas públicas se reflita em ações que gerem benefícios sociais. A partir desse momento torna-se imperativo conhecer as interações geradas pelo Participa.br e entender como o governo e as organizações públicas ou privadas se beneficiaram desse novo instrumento de comunicação.

Nesse cenário, a análise de redes sociais toma força e torna-se um instrumento que viabiliza a análise dos elementos e atores que interagem no processo de formulação de políticas públicas, assim como as relações que emergem dessa interação, oferecendo informações relevantes para a elaboração de estratégias destinadas ao fortalecimento da ação coletiva entre os diferentes agentes envolvidos. Essa abordagem permite compreender como as estruturas sociais e os padrões de relações podem influenciar no processo e direcionar os resultados obtidos.

Sendo assim, este estudo visa analisar o módulo "consulta pública" da rede social Participa.br utilizando como fonte de informações os dados disponíveis na ferramenta. Mesmo sabendo que as consultas são participações individuais e voluntárias, os dados foram agrupados pelas organizações as quais autor da contribuição é vinculado, com intuito de responder a seguinte pergunta de pesquisa "Qual a influência das organizações de TI na elaboração de políticas públicas voltadas para o governo eletrônico utilizando como base o Participa.br?”. 
Esse artigo está estruturado da seguinte forma: além desta seção, que apresenta cenário, motivação, especificação do problema, a pergunta de pesquisa e a organização do estudo, existem outras três seções, organizadas conforme se descreve a seguir.

A Seção II apresenta a metodologia de pesquisa aplicada ao artigo, a forma de coleta de dados, dando uma visão geral do que é a plataforma Participa.br e de como os dados estão estruturados na mesma. Além disso, detalha como os dados da plataforma foram transformados para elaboração do estudo. A Seção III apresenta quais métricas foram utilizadas e as análises dos dados tomando como base tabelas e grafo gerados pela ferramenta Gephi (ferramenta escolhida para dar suporte às análises da pesquisa). A

Seção IV oferece as conclusões desta pesquisa, demonstrando suas contribuições, limitações e trabalhos futuros.

\section{Metodologia de Pesquisa}

Em função dos objetivos, esta pesquisa é classificada como descritiva, utilizando a estratégia de estudo de caso. Neste estudo foi usada a pesquisa descritiva que, segundo Vergara (2014), tem como objetivo a descrição das características de determinada população, fenômeno ou o estabelecimento de relações entre as variáveis.

\subsection{Coleta dos Dados}

Para melhor contextualizar a coleta de dados esta seção descreve um pouco o que é o Participa.br e como seus dados estão estruturados. A Figura 1 apresenta a tela inicial do Portal.
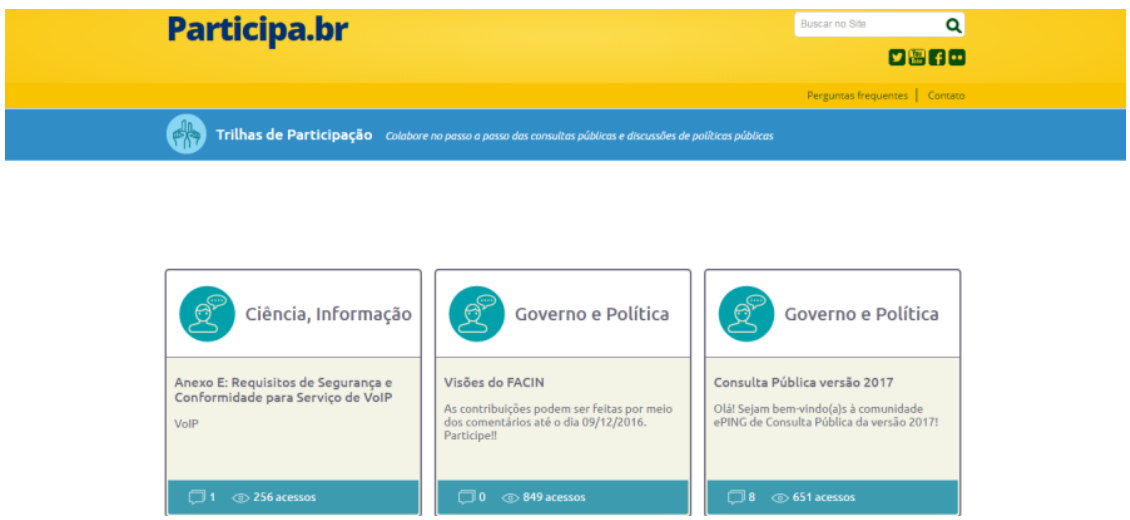

Figura 1 - Tela Inicial da Ferramenta Participa.br

O Portal da Participação Social é uma plataforma virtual voltada à construção de um conjunto de ambientes que poderão ser utilizados por gestores e servidores para proporcionar novas formas de participação a serem apropriadas pela cidadania e à contextualização, organização e facilitação do acesso do cidadão às formas de incidir nas políticas públicas do governo brasileiro, objetivando servir como um repositório agregador do conhecimento sobre participação social disperso na rede. Os Usuários terão acesso a ferramentas de comunicação e interação, fóruns de debate, chat, vídeos, mapas, trilhas de participação com mecanismos de consulta [BRASIL 2014].

Para o contexto deste estudo a trilha "consulta pública" será objeto da análise, visto que se pretende analisar a construção colaborativa de políticas públicas. Apesar da 
consulta pública ser um instrumento de construção de conhecimento colaborativo individual, esta pesquisa optou por obter informação da organização vinculada a cada autor de sugestão submetida, além disso outro dado preponderante para pesquisa foi a identificação de qual tópico disponibilizado para debate público, entende-se por tópico: Guias, Framework Corporativo, Políticas Públicas, Ações e Programas Estratégicos do Governo Federal.

A Figura 2 mostra o modelo conceitual da Plataforma.

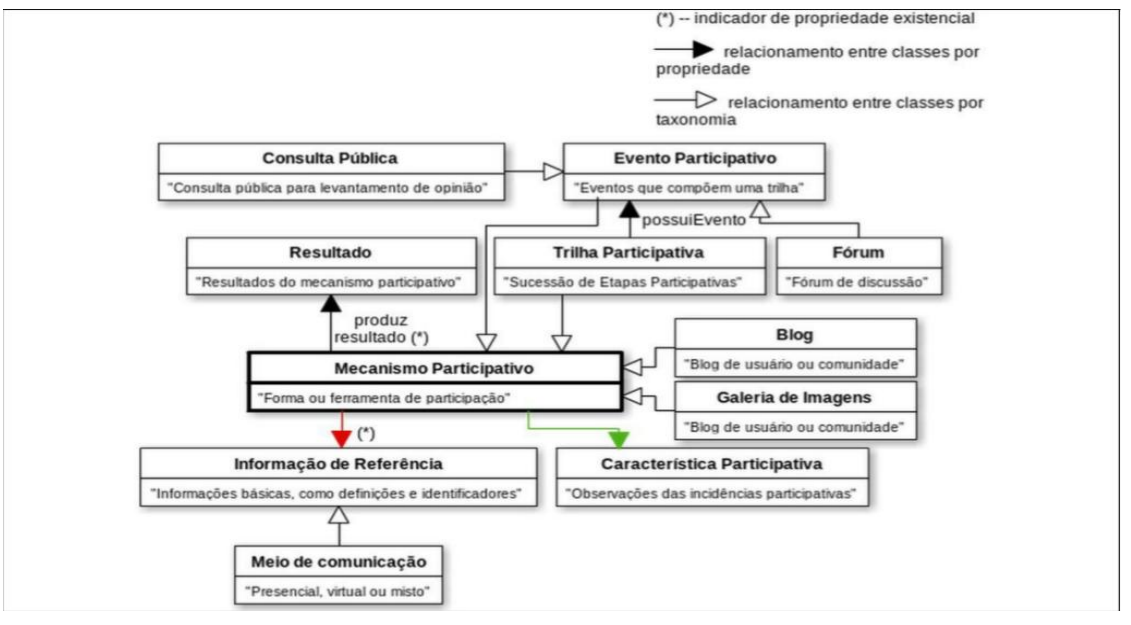

Figura 2 - Modelo Conceitual - Fonte: [FABRI 2014 apud MEIRELIES 2014]

Os dados coletados seguem ao diagrama apresentado na Figura 3, onde as informações das consultas públicas são armazenados utilizando as classes $\{$ ConsultaPublica $\},\{$ Item $\},\{$ Sugestao $\},\{$ Autor $\}$ e $\{$ Empresa $\}$. O arquivo recebido para elaboração da pesquisa foi no formato csv(Comma-Separated Values) .

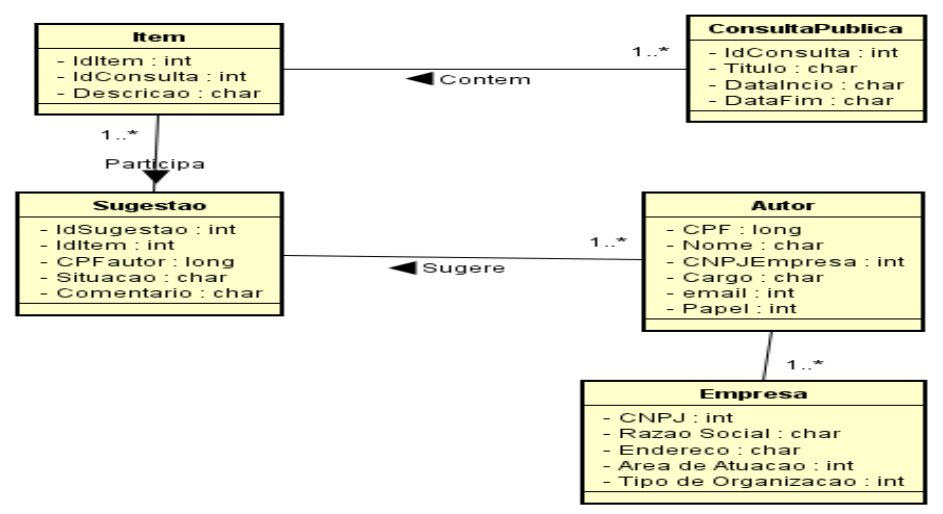

Figura 3 - Diagrama das Classes Vinculadas a Consulta Pública do Participa.br

\subsection{A Transformação dos Dados}

A transformação dos dados seguiu a seguinte sequência: a) eliminar os dados de baixa relevância para preparação do ambiente analítico; b) assegurar que as informações pessoais fossem preservadas; c) identificar dados inconsistentes; e d) colocar os dados no formato que a ferramenta reconheça.

Dessa forma, os dados foram carregados da seguinte maneira: informações contidas na classe $\{$ Sugestao\} passaram a integrar a Tabela Aresta onde, quando a 
situação estava preenchida com "0" (não aceito) o atributo "Source" foi preenchido com Identificador do Autor e o atributo "target" preenchido com Identificador da Consulta, para o caso da situação igual a "1" (aceito) o atributo "Source" foi preenchido com Identificador da Consulta e o atributo "target" preenchido com Identificador do Autor. Para preencher a Tabela Nó utilizou-se Identificador do Autor e Nome da Organização. A ferramenta Gephi ${ }^{2}$ foi o software escolhido para gerar a estrutura da rede, o grafo de distribuição da rede e a visualização dos graus de entrada e saída. A Figura 4 mostra um extrato das tabelas preenchidas.
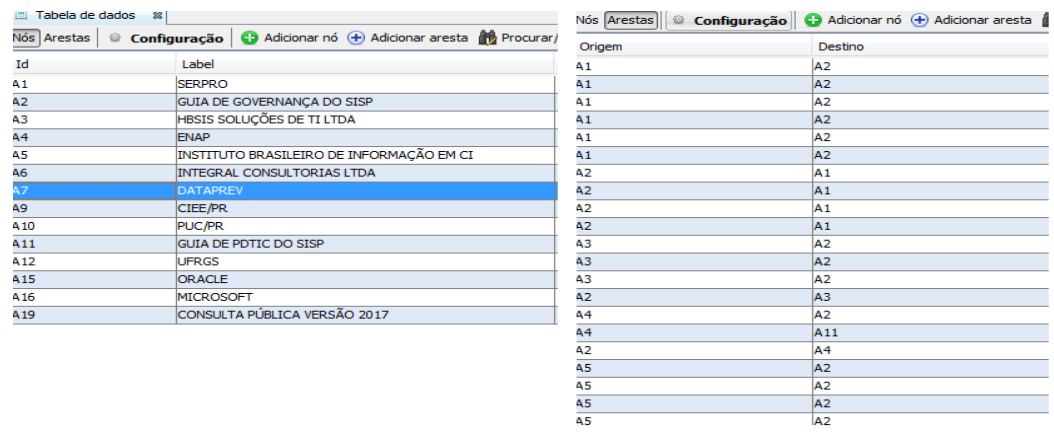

Figura 4 - Tabelas Nós e Aresta Fonte: Autor gerado no Software Gephi

Ainda em relação aos dados utilizados nesta pesquisa, estão computadas as informações referentes à consulta pública para o Guia de Governança de TIC do SISP (modelo referencial de governança de TIC do SISP $^{3}$, para que órgãos e entidades do Sistema desenvolvam e aperfeiçoem a governança de TIC em suas instituições [MPOG 2015a]); Guia de PDTIC ${ }^{4}$ do SISP (tem por finalidade disponibilizar conhecimento para auxiliar a elaboração e o acompanhamento de um PDTI, para aprimorar a gestão da TI nos órgãos da Administração Pública Federal - APF[MPOG 2015b]); e Consulta Pública versão 2017 (conhecida como ePING ${ }^{5}$ define que, sempre que possível, serão adotados padrões abertos nas especificações técnicas [BRASIL 2017]). Acrescenta-se que por termos informações apenas de três consultas, o volume de dados é relativamente pequeno.

Em relação aos indicadores de rede utilizados no estudo destacam-se o grau de entrada (é a soma das interações que os nós têm com os outros - $\sum \mathrm{k}$ (in)) e o grau de saída (é a soma das interações que os nós têm com os outros - $\sum \mathrm{k}$ (out)). Para verificar a influência de um determinado nó dividiu-se o grau de entrada pelo grau de saída.

Equação - Grau de Influência é: $\sum \mathrm{k}$ (in)/ $\sum \mathrm{k}$ (out)

\footnotetext{
2 A ferramenta para manipulação de grafos mais utilizada tem sido o Gephi, um software livre colaborativo mantido por um sediado na França, com inúmeras aplicações em áreas como as ciências biológicas ou a economia - aqui, comentamos sua utilização em projetos ligados à história e à análise textual.

3 Sistema de Administração dos Recursos de Tecnologia da Informação - SISP

4 Plano Diretor de Tecnologia da Informação e Comunicação - PDTIC

5 Padrões de Interoperabilidade de Governo Eletrônico - ePing
} 


\section{Análise de Resultados}

A primeira análise das consultas públicas do Participa.br é feita apresentando a distribuição do grau de entrada e saída agrupando as sugestões enviadas pelos autores por instituição vinculada, como pode ser observado na Figura 5 apresentada abaixo.

\begin{tabular}{|c|c|c|c|c|c|}
\hline \multicolumn{6}{|c|}{ (10) Tabela de dados $\&$} \\
\hline \multirow{2}{*}{$\begin{array}{l}\text { Nós Arestas } \\
\text { Id }\end{array}$} & \multirow{2}{*}{$\frac{\text { Configuração }}{\text { Label }}$} & \multirow{2}{*}{$\begin{array}{l}\text { I aresta } \\
\text { Interval }\end{array}$} & \multirow{2}{*}{$\begin{array}{l}\text { Procurar/Substituir } \\
\text { Grau de entrada }\end{array}$} & \multicolumn{2}{|c|}{ Importar planilha 윤 } \\
\hline & & & & Grau de saída & Grau \\
\hline A1 & SERPRO & & 19 & 29 & 48 \\
\hline A2 & GUIA DE GOVERNANÇA DO SISP & & 58 & 29 & 87 \\
\hline A3 & HBSIS SOLUÇÕES DE TI LTDA & & 2 & 6 & 8 \\
\hline A4 & ENAP & & 6 & 11 & 17 \\
\hline A5 & INSTITUTO BRASILEIRO DE INFORMAÇÃO EM CI & & 2 & 8 & 10 \\
\hline A6 & INTEGRAL CONSULTORIAS LTDA & & 4 & 7 & 11 \\
\hline A7 & DATAPREV & & 9 & 14 & 23 \\
\hline A9 & CIEE/PR & & 2 & 5 & 7 \\
\hline$A 10$ & PUC/PR & & 4 & 8 & 12 \\
\hline A11 & GUIA DE PDTIC DO SISP & & 26 & 14 & 40 \\
\hline A12 & UFRGS & & 1 & 3 & 4 \\
\hline A15 & ORACLE & & 1 & 2 & 3 \\
\hline A16 & MICROSOFT & & 1 & 2 & 3 \\
\hline A19 & CONSULTA PÚBLICA VERSÃO 2017 & & 11 & 8 & 19 \\
\hline
\end{tabular}

\section{Figura 5 - Apresenta a distribuição dos graus de entrada e saída das contribuições das organizações. Fonte: Autor}

Observando a Figura 5 e levando em consideração que as consultas públicas foram feitas para: Guia de Governança de TIC do SISP, Guia de PDTIC do SISP e Consulta Pública versão 2017; é possível constatar que os empregados de SERPRO ${ }^{6}$, DATAPREV $^{7}$ e ENAP $^{8}$ são os que enviam mais sugestões (grau de saída) para os temas. Para sugestões aceitas (grau de entrada), também são dessas organizações os autores com maior número de aceitação, fato natural, já que esses órgãos têm suas linhas de negócios alinhados aos entes públicos federais.

Tabela 1 - Grau de Influência das organizações na política pública

\begin{tabular}{|c|c|c|c|}
\hline Organização & $\begin{array}{l}\text { Grau de Entrada } \\
\sum \text { k (in) }\end{array}$ & $\begin{array}{l}\text { Grau de Saída } \\
\sum \text { k (out) }\end{array}$ & $\begin{array}{l}\text { Grau de Influência } \\
\sum \mathbf{k} \text { (in) } / \sum \mathbf{k} \text { (out) }\end{array}$ \\
\hline SERPRO & 19 & 29 & 0,655 \\
\hline DATAPREV & 9 & 14 & 0,642 \\
\hline ENAP & 6 & 11 & 0,545 \\
\hline PUC/PR & 4 & 8 & 0,500 \\
\hline INTEGRAL CONSULTORIAS LTDA & 4 & 7 & 0,571 \\
\hline \begin{tabular}{lcccc} 
INSTITUTO & \multicolumn{2}{c}{ BRASSILEIRO } & DE \\
INFORMAÇÃO & E & EM & CIÊNCIA & DA \\
INFORMAÇÃO & & & & \\
\end{tabular} & 2 & 8 & 0,25 \\
\hline HBSIS SOLUÇÕES DE TO LTDA & 2 & 6 & 0,333 \\
\hline CIEE/PR & 2 & 5 & 0,400 \\
\hline UFRGS & 1 & 3 & 0,333 \\
\hline ORACLE & 1 & 2 & 0,500 \\
\hline MICROSOFT & 1 & 2 & 0,500 \\
\hline
\end{tabular}

\footnotetext{
6 Serviço Federal de Processamentos de Dados - SERPRO

7 Empresa de Tecnologia e Informações da Previdência Social

${ }^{8}$ Fundação Escola Nacional da Administração Pública - ENAP
} 
Analisando com um pouco mais de detalhe é possível inferir outras variáveis que corroboram para a participação de pessoas ligadas as instituições na construção de políticas, primeiramente esses indivíduos, invariavelmente, estão engajados em projetos que serão impactados por tais políticas e, como possuem conhecimentos sobre o tema, têm interesse individual em externar suas sugestões, já o segundo ponto diz respeito aos compromissos dessas instituições com a sociedade, visto que duas são empresas públicas federais e a outra fundação federal.

A Figura 6 apresenta um grafo da rede da interação das organizações com as políticas públicas.

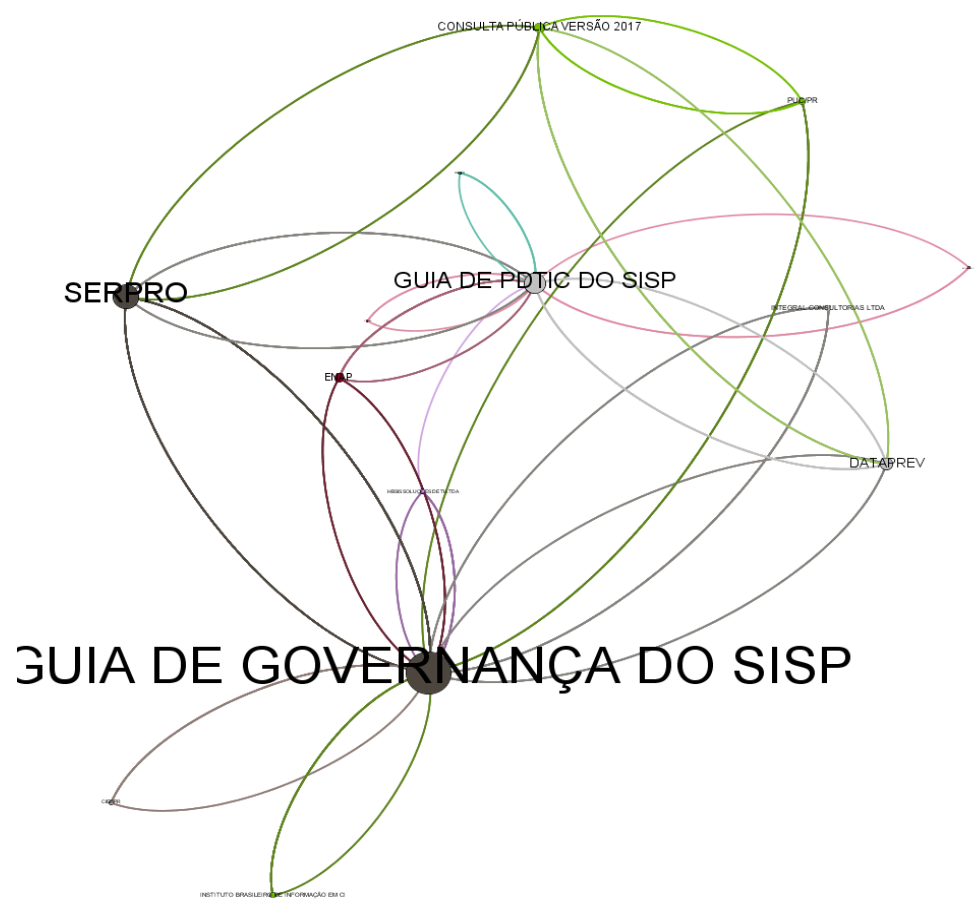

Figura 6 - Grafo gerado das interações das organizações com as políticas públicas. Gerado pela ferramenta Gephi.Fonte: Autor

Ao analisar o grafo apresentado pela Figura 6 fica mais evidente a influência dessas instituições na construção de políticas públicas que se relacionam com suas linhas de negócios. O SERPRO é uma instituição muito atuante contribuindo para elaboração de políticas pública voltada para governo eletrônico mesmo quando a contribuição parte de uma iniciativa individual do seu corpo funcional, não tão distante as contribuições de empregados da DATAPREV ou da ENAP também demonstram ser consistentes na influência das políticas públicas de interesse desses órgãos.

O interesse do SERPRO, DATAPREV e ENAP sobre os três temas de consultas públicas pesquisadas tem um motivo, vejamos: a) a Instrução Normativa 04/2010 [MPOG 2010] determina que "As contratações de que trata esta instrução normativa deverão ser precedidas de planejamento, elaborado em harmonia com o PDTI, alinhado à estratégia do órgão ou entidade."; b) a mesma instrução normativa em seu capítulo II, artigo $10^{\circ}$, ainda em relação as contratações de serviços de TI, acrescenta que deve as 
contratações estar atentas "observância às políticas, premissas e especificações técnicas definidas pelo e-PING e Modelo de Acessibilidade em Governo Eletrônico - e-MAG".

Contudo, a IN 04/2010 abre uma brecha para contratações de serviços de TI onde o contratado seja o SERPRO, não há necessidade de um PDTIC. Segundo Fagundes (2011) a ENAP desenvolveu o modelo de referência de PDTI, utilizado na SLTI $^{9}$ no Programa Desenvolvimento de Gestores de Tecnologia da Informação PDGTI ministrado pela ENAP. Já a DATAPREV foi responsável pela elaboração do PDTIC do INSS ${ }^{10}$ e do Ministério da Previdência Social, além de ser responsável pela Governança e Gestão de TI para infraestrutura e serviços de TI desses órgãos [TCU 2015] .

Tais informações corroboram para a estrutura de relacionamento da rede gerada pelo grafo, visto que essas organizações têm interesses nas políticas públicas relacionadas ao governo eletrônico o que ficou evidenciado no grafo de interações. Contudo, não se pode deixar de mencionar que as sugestões são de cunho individual.

\section{Conclusão}

Este artigo apresentou a rede formada pelas Consultas Públicas do Guia de Governança de TIC do SISP, Guia de PDTIC do SISP e e-Ping versão 2017 e os autores de sugestões para essas consultas. Por meio de técnica de análise da rede social foi possível a identificação de características de associação e a influências de empresas do ramo de TI ou entidades governamentais de interesse comum em uma determinada política pública.

A primeira análise evidenciou que, mesmo as participações com sugestões e comentários para uma determina consulta pública sendo uma iniciativa voluntária e individual, o vínculo dos autores com organizações que, por força de sua linha de negócio e seus projetos, naturalmente influenciam do indivíduo a externar sua opinião, bem como indiretamente influenciam no debate sobre uma determinada política pública.

Outra forte evidencia encontrada na análise é o grande número de indivíduos ligados a organizações como SERPRO, DATAPREV e ENAP que sentirem-se a vontade para participar do debate sobre o tema. Esse conforto é gerado por tais empregados estarem vinculados a projetos e ações que são impactadas direta ou indiretamente pela política pública em questão, visto que o conhecimento adquirido por esses indivíduos os levam a ter clareza sobre os impactos que a política pode gerar na execução dos seus trabalhos e nas relações geradas por essa ação governamental. Desta forma, seus comentários e sugestões têm maior probabilidade de serem aceitos pela comunidade que avalia a contribuição.

Nesse contexto, outra leitura das relações geradas pelo Participa.br é a constatação que SERPRO, DATAPREV e ENAP influenciam bastante as políticas públicas voltadas para o segmento de governo eletrônico, visto que as três organizações apresentaram grau de entrada e saída, o que detona viabilizar políticas que vão ao encontro de seus interesses.

\footnotetext{
${ }^{9}$ Secretaria de Logística e Tecnologia da Informação - SLTI
}

10 Instituto Nacional de Seguridade Social 
As constatações que esse estudo levou foco não fogem do senso comum antes percebido, pois são instituições públicas que trabalham para agentes públicos e, muitas vezes, beneficiam-se da boa relação para influenciar políticas e, consequentemente, o mercado no que diz respeito a governo eletrônico.

Entretanto, outra evidência apresentada pela análise é o baixo número de indivíduos com interesse em debater sobre políticas ligadas a TI, a oportunidade de debater políticas públicas é um clamor social, porém quando existe um espaço essa interação não acontece no volume desejável. Deve-se refletir sobre como o canal comunicação foi aberto, quais mecanismos foram utilizados para facilitar a participação popular, como viabilizar o desejo por um envolvimento do cidadão.

É louvável que um novo espaço seja criado para aproximar governo e sociedade, porém esse espaço precisa evoluir para que o ambiente participativo seja bem aproveitado gerando políticas eficazes, eficientes e efetivas.

De forma geral, a análise de rede social mostrou-se uma alternativa apropriada para a investigação das relações de geradas pela plataforma Participa.br, podendo ser aplicada em maior escala para estudos de comunicação científica, assunto de grande interesse para a ciência da informação.

\subsection{Limitações}

Este estudo foi feito utilizando informações de apenas três consultas públicas o que inviabilizou uma pesquisa mais aprofundada da rede do Participa.br. O volume de dados disponibilizado para análise da pesquisa foi pequeno não permitindo analisar as propriedades estruturais da rede tais como: coeficiente de agrupamento, geodesia espacial, identificação de cliques, distribuição gráfica dos graus. Aliado a isso, o estudo não verificou a rastreabilidade em prováveis decisões finais. Desta forma, o estudo se ateve a responder a pergunta de pesquisa.

\subsection{Trabalhos Futuros}

Elaborar análise das propriedades estruturais das redes levando em consideração todas as ferramentas de comunicação e interações contidas no ambiente Participa.br, ampliar o escopo de pesquisa considerando características espaciais de cada membro que postar informações na Plataforma, bem como fazer um estudo do comportamento da rede em cada um dos seus ambientes de interação, considerando as vantagens e desvantagens da utilização do ambiente Participa.br.

\section{Referências}

Brasil, Presidência da República (2013) "Lançamento do novo Portal Brasil", http://www.brasil.gov.br/governo/2013/09/dilma-anuncia-reformulacao-do-portalbrasil, fevereiro.

Brasil (2014) 'Portaria $\mathrm{n}^{\mathrm{o}} 36$ de 11 de novembro de 2014', Diário Oficial da União. Brasília - DF, quarta-feira, 12 de novembro de 2014. Disponível em: http://pesquisa.in.gov.br/imprensa/jsp/visualiza/index.jsp?data=12/11/2014\&jornal=1 \&pagina $=1 \&$ totalArquivos $=92$. 
BRASIL (2017) Portal de Participação Social, Consulta Pública ePing versão 2017. Disponível em: http://www.participa.br/e-ping/consulta-publica-versao-2017/parte-ivisao-geral-da-eping.

FAGUNDES, V. (2011) "PDGovTI Modelo de Plano Diretor de Tecnologia da Informação Baseado em Arquitetura Corporativa e Governança de TI em Governo", Dissertação de Mestrado - Programa de Engenharia de Sistemas e Computação UFRJ.

MEIRELLES, Paulo R. M. (2014) "Documento com proposta de extensão de ontologia para o Portal de Participação Social, dados de Conferências Nacionais e Conselhos Nacionais, incluindo propostras de articulação entre os dados desses instrumentos". Disponível em: http://biblioteca.participa.br/jspui/bitstream/11451/1120/1/PRODUTO $\% 2006 \% 20 \mathrm{PR}$ ODOC\%20PNUD\%20\%20ONTOLOGIA\%20PARA\%200\%20PARTICIPA.BR.pdf.

MPOG, Ministério do Planejamento, Orçamento e Gestão (2015a) “Guia de Governança de TIC do SISP MPOG”, Brasília.

MPOG, Ministério do Planejamento, Orçamento e Gestão (2015b) “Guia de PDTIC do SISP MPOG”, Brasília.

MPOG, Ministério do Planejamento, Orçamento e Gestão. (2010) "Secretaria de Logística e Tecnologia da Informação. Instrução Normativa SLTI $n^{\circ}$ 4, de 12 de novembro de 2010". Disponível em: http://www.comprasgovernamentais.gov.br/paginas/instrucoes-normativas/instrucaonormativa-no-04-de-12-de-novembro-de-2010.

TCU, Tribunal de Contas da União (2015) “TC 005.693/2015-2”. Disponível em: www.tcu.gov.br/Consultas/Juris/Docs/judoc/Acord/.../AC $071610 \quad 16$ P.doc.

SOARES, F. de M. (1997) "Direito Administrativo de Participação (Cidadania, Direito, Estado e Município)", Belo Horizonte: Del Rey.

VERGARA, S. C. (2004) "Projetos e relatórios de pesquisa em Administração", São Paulo: Atlas. 